\title{
Building Bridges: Strategies to Overcome Challenges in Social Work with Immigrants and Refugees
}

\author{
By Justin S. Lee ${ }^{*}$ \& Suzie Weng ${ }^{\dagger}$
}

Social work practitioners and researchers must overcome several barriers to improve access to immigrant and refugee populations. The intent of this paper is to present both challenges and strategies that impact practice and research with members of historically underrepresented immigrant and refugee groups. Though not the original intent of the research projects, we identified these challenges and strategies during two studies that we conducted. Challenges and strategies are set in the context of UyBico and colleagues' (2007) framework-culture, individual, structure, and institution. We advocate for increased outreach and collaboration with minority newcomers, and a more coherent link with the social work profession.

Keywords: access, engagement, immigrants, newcomers, refugees, social work.

\section{Introduction}

As the immigrant and refugee minority population in United States continues to increase, there is a growing recognition of the need to include these ethnic minorities in social work practice and research. While the issue of minority participation has received increased attention recently, few studies directly address the challenges in access in social work research and practice. Current literature on overcoming access challenges tends to focus on clinical studies or in health research that includes randomization and comparison groups. While that may be helpful, social work researchers and practitioners need to access minorities outside of clinical studies.

In this paper, we address two questions: First, as social workers, how should we understand the challenges of access to immigrant and refugee populations? Second, how should we use the strategies available to overcome these challenges? We identify several examples of challenges and strategies in existing literature, and many we learned through conducting two studies that we will use to illustrate our findings. Following the brief description of our two studies, we present UyBico et al. (2007) framework. This will help us understand how to think about challenges at the cultural, individual, structural, and institutional level, and strategies to address these challenges at each level. To be clear, the challenges and strategies that we identify in this paper were a secondary finding, and not the primary original intent of the studies. For this

\footnotetext{
*Assistant Professor of Social Work, Idaho State University, USA.

${ }^{\dagger}$ Assistant Professor of Social Work, California State University, Long Beach, USA. 
reason, we have kept the study description concise and relevant to the question at hand.

We will define some of the terms we use from the outset. 'Access' includes the ability of immigrants and refugees to access services, as well as social work practitioners' and researchers' ability to engage with immigrants and refugees. The terms 'immigrants', 'refugees', and 'ethnic minorities' are used somewhat interchangeably for the purposes of this paper, though we recognize there are important differences between and within these categories. We chose to use the term 'challenge' rather than its common synonym 'barrier' because 'challenge' was a common descriptor used by participants in both studies, though we can treat them as interchangeable for our purposes. We use the term 'strategies' in reference to any means that can be utilized to overcome challenges.

\section{Brief Description of Study Examples}

\section{Ethnic Agencies Founders}

The first author examined how individuals created ethnic agencies and programs to address unmet community needs. This study built on Jenkins' (1980) seven characteristics of an ethnic agency: 1) serving primarily ethnic clients; 2) staffed by a majority of individuals who are of the same ethnicity as the client group; 3) an ethnic majority on its board; 4) ethnic community and/or ethnic power-structure support; 5) integrating ethnic content into its programs; 6) viewing strengthening the family as a primary goal; and 7) maintaining an ideology that promotes ethnic identity and ethnic participation in decision-making processes. Intent, implementation, and experience of the founders in their development of ethnic agencies were explored. The IRB approved study used purposive sampling with the goal of maximum variation (Flick 2006) and included five people who founded ethnic agencies. Challenges experienced by the researcher are incorporated into the paper and referred to throughout as "the founders' study."

\section{Unaccompanied Refugee Minors}

The second author studied unaccompanied refugee minors (URMs) who enter the program in the U.S. through The State Department which gives a small number of refugee visas each year and contracts with two national faith-based organizations to provide for their basic needs. URMs are placed in one of 14 states that have programs established to help them transition into adulthood with the expectation that they will be eligible for permanent citizenship. This study focused on developing a theory of culturally relevant success from the perspective of former URMs. Upon successful ethical review (IRB), 15 participants were interviewed with sampling for maximum variation. In the following sections, we will refer to the "URM study" as 
challenges and strategies for improving future engagement with refugee populations are incorporated into the narrative.

\section{Access Related Challenges}

In this section we combine access challenges in our two studies just mentioned and in the broader literature. The challenges in accessing and outreach to hard-to-reach, invisible, or rare populations are well established. Among ethnic minority populations, Asian Americans and Latino Americans may be considered a rare population - those that lack adequate sampling frames - due to its undocumented individuals. Asian Americans may be somewhat invisible because they are often not considered in discussions of community needs or service interventions. The rarer the population, the more potential time and other resources it will require to engage. Consequently, it is more difficult for social workers to try to work with these populations.

Several reasons exist as to why immigrants and refugees may be unwilling to utilize social services or participate in social science research. Access related barriers can be categorized as cultural, individual, structural, and institutional (UyBico et al. 2007). Cultural barriers include reverence for authority, acculturation, cultural differences between target population and social worker, and stigma. Individual barriers include mistrust of research and government, and familism. Structural barriers include logistics in participation and engagement, the complexity of services systems and research, and the small size of immigrant and refugee groups. Institutional barriers include social worker biases and stereotypes. This section uses this framework to describe access challenges identified in the literature and those faced by the researchers.

\section{Cultural Challenges to Access}

Social workers must be attuned to cultural characteristics that may influence ethnic minority participation so as to avoid inadvertently manipulating populations that are already vulnerable. Reverence for authority, where persons of power are respected for their knowledge (Brugge et al. 2005), is a cultural factor for many groups that can lead to individuals being more likely to engage at the suggestion of someone who is well-respected. Level of acculturation may also be a factor because Asian Americans who were more acculturated into American society have been found to less likely participate in research (Brugge et al. 2005). The target population and social workers may differ in terms of socioeconomic status, education, language, and/or ethnicity. These differences have consequences as to how social workers and target population view the purpose and importance of research or service. Additionally, ethnic minorities are more likely to be misdiagnosed when it comes to psychotic disorders and depression than Whites (Jones and Gray 1986). Finally, sense of shame to 
the family is avoided so as not to embarrass the family, resulting in less participation in stigmatized issues like mental health and substance abuse.

In the URM study, former refugees had learned English, become adept at following normative behavior in the U.S., and seemed to have acquired many cultural values. However, one challenge had to do with scheduling in which for many of the participants, time was a fluid concept that led to arriving late or not showing up to a scheduled interview. They had adapted to the western ideal of keeping an extremely full schedule and many seemed torn between cultural group membership and their newly acquired individualism.

In the founders study, the cultural challenges were related to gender in that all the participants and a majority of potential participants were female. Because ethnic agencies are a fairly new phenomenon, a sampling frame of founders of these agencies does not exist. Therefore, it is hard to know the gender breakdown of the founders. With many Asian cultures being a patriarchal society (Hanssen 2004), that may influence men focused on supporting the family rather than starting a nonprofit with little financial benefit.

\section{Individual Challenges to Access}

Access related challenges at the individual level include familism and mistrust. Familism, decision-making by the family (Brugge et al. 2005), may mean that individuals are more likely to participate if the family decides to do so or at another family member's encouragement. The influence of familism is against the western value of autonomy in which freedom of choice and self-determination is central to decision making. In collectivist Asian and Latin cultures, family members are key players in the decision making process and decisions are made based on the interests of the family over the individual.

Individuals may be skeptical of their participation due to historical mistreatment of ethnic minorities in research (Alvidrez and Arean 2002). In clinical research, African, Latino, and Native Americans have expressed their mistrust of medical institutions and concerns of being used as guinea pigs (Roberson 1994). One reason for ethnic minority skepticism of research is the historical research supporting racist ideology to show minorities are inferior in order to justify slavery and discrimination (Bhopal 1997). Mistrust about research continues among ethnic minorities despite increased protections in the last few decades (Roberson 1994). Social workers can be seen as another representative of the formalized service system.

In the study with URMs, many potential participants were mistrustful of researchers, perhaps because they feared that their stigmatized status as refugees would be exposed. Prior to emigrating, several participants were told to mistrust the government and the people in the U.S. However, upon arrival in the U.S., often the first advice they received was to assimilate, adopting American norms and values and leaving their culture of origin in the past. This resulted in some who agreed readily while others were 
skeptical of the study as well as a variety of responses about their service experience. One challenge in particular was that participants seemed to feel like the research was intended to assess the quality of care they had received rather than the intent of learning about their emigration experience. The responses, therefore, were often geared toward their appreciation for services provided for them from their host agency rather than a genuine critique of their overall experience.

\section{Structural Challenges to Access}

Immigrants and refugees tend to be more low income and struggle with issues of day-to-day survival. Low-income immigrants may incur additional expenses or miss earning opportunities if they seek services or participate in research. Elders may be hesitant if they are responsible for the care of their grandchildren. Undocumented immigrants may fear deportation, particularly if social workers involved are representatives of government agencies. These groups may not comprehend the complexity of the service delivery system or research projects. The unfamiliarity may also contribute to mistrust of social workers attempting to improve the system.

The participants in the founders study were immigrants and refugees but they were not struggling for day-to-day survival. They were educated professionals who saw a need in their own communities and decided to take action in addressing them by starting agencies that are ethnic specific to their community. Part of the difficulty with recruiting the founders is their availability to participate in the research due to their existing community involvement. Another challenge is that the participants recruited were geographically spread out, which made it difficult for the interviews to take place in person.

In the founders study, the recruitment process was extremely difficult and is reflected in the small sample size. The small number in ethnic minority populations has made social work research and practice difficult, making it harder to justify funding for studies or for available services. Current literature on immigrants and refugees is often based on a few subgroups in geographical areas where a majority reside. The gaps in research indicate that aspects of some immigrant groups are understudied. Because of the limited practice and research on ethnic groups, crossculturally valid interventions, diagnostic procedures, and research are also limited, which results in an appropriate baseline of knowledge and theory may not exist (Sue and Sue 2003). Lack of meaningful knowledge means researchers, policymakers, and practitioners do not have a good understanding of minority populations. This may lead to inadequate and ineffectual provision of services. To overcome the small population numbers, researchers have aggregated different groups into one. This lumping may lead to stereotypes and overgeneralization in social work practice.

In the URM study, the participants were adults who had received services while minors because of the structural barrier of the service 
provider. The agency was the primary legal guardian and refused to have their service recipients participate in research activities. Given their status as underage refugees, this is in part understandable, but it leaves a glaring question: How are promising practices developed with an unreachable population?

\section{Institutional Challenges to Access}

Social workers' own attitudes may contribute to the difficulty of accessing ethnic populations (Sheikh et al. 2009). They may hold actual or potential biases such as racism or fears. They may also believe in stereotypes and prejudices about the difficulties of engaging with ethnic minorities. If these biases are not addressed, social workers' attitudes can be communicated through their interactions. Social workers, including those who are ethnic minorities, are often educated and trained in a dominant perspective. In the United States' pluralistic society, that dominant perspective may not be shared in the communities in which social workers find themselves.

Some of the Asian cultural traits may have contributed to Asian Americans being regarded as a model minority by the dominant society. The myth refers to the assumption that all Asian Americans achieve universal and unparalleled academic, occupational, socioeconomic, and general success (Museus and Chang 2009). The stereotype gives some the excuse that Asian Americans do not need attention and not to include them in service provision, research studies, or public discourse - in effect, subjugating their voices and rendering them invisible. Aggregated data may show Asian Americans to outperform other racial and ethnic groups but more detailed stratified data of its subgroups show that some are in desperate need of services.

In addition to personal biases of social workers, the URM study found that the institutional norm favored assimilation of refugee adolescents rather than accommodation of cultural difference, an alternative shown to be healthier for both physical and mental health. Additionally, Derluyn and Broekaert (2008) point out, there is a glaring difference between the legal and psychological approach to refugee migration. These URM teens seemed to be reluctant to say too much in criticism of the U.S. since their legal immigration status was not maintained by the same agency that provided them with basic services. This reluctance, combined with the difficulty in separating the research study from the agency from which they received services, impacted their responses.

\section{Strategies/Lessons Learned About Access}

This section explores strategies found in the literature to overcome the cultural, individual, structural, and institutional challenges social workers may face when attempting to access immigrant and refugee groups for 
practice or research. This section also includes lessons learned by the authors who conducted research on founders of ethnic agencies and URMs.

In general, social workers are successful in engaging individuals for several reasons. For some members of minority groups, personal benefit is their central motivation. Brown and Topcu (2003) found minorities are willing to be part of health research if asked. For others, altruism emphasizes the potential to help others or benefit society. In O'Brien and associate's (2006) study, a majority of minorities, aside from African Americans, were willing to participate in clinical research if it was valuable to their community. In the founders study, for example, one founder was willing to participate because the findings may be helpful to others who also wished to start ethnic-specific services for their community. In the URM example, participants were anxious to "give back" to their community that supported them during their transition. They also saw participation in the study as a responsibility to improve the program for others that would follow. The combination of duty and sense of community was likely augmented by the close personal relationship that the agency social worker - the person asked to recruit research participants- had with them.

\section{Cultural Strategies to Increase Access}

To overcome some of the cultural challenges mentioned above, several strategies have been identified. Smith (2009) suggests gaining the trust of the immigrant and refugee population, social workers being upfront and transparent about intentions was one way to gain participant buy-in. Because of the many differences within racial and ethnic groups, social workers must be knowledgeable about which groups are in need of services or advances in the knowledge base. For Asian Americans, physicians are considered an important authority figure (Chen et al. 2005). One strategy to reduce the stigma is by applying a medical model to explain psychiatric illnesses (Alvidrez and Arean 2002, Chen et al. 2005). Physician referrals have been found to be an effective recruitment strategy (Epstein 2008).

Intensive outreach activities, whether for practice or research, may be necessary with some communities. For social workers focused on an issue that may be stigmatized by the ethnic community, an educational campaign must be part of the recruitment process. Presentations can be given at various community events. Culturally appropriate brochures can be provided at local restaurants and shops. The ethnic community newsletter or paper can be used. For Asian American elders with mental health needs, newspaper articles written in the patient's voice was most effective (Chen et al. 2005). In larger communities, local ethnic radio programs may reach those who have lower literacy levels and may be played at locations where there is a dense ethnic population such as places of employment or service providers who target ethnic clients. Leslie (1992) found announcements of the project at English as second language classes and door-to-door canvassing in a heavily populated Hispanic population to be successful strategies. In the founders study, the researcher had friends who served as 
gatekeepers in recruiting founders who worked on issues that are stigmatized by the community. For non-stigmatizing issues related to social work practice, gatekeepers can also be used for outreach purposes (Feldman et al. 2003).

When attempting to access hard-to-reach populations, whether to conduct research or outreach to provide services, multiple approaches may be necessary and was found to be the most effective (UyBico et al. 2007). Coe et al. (2008) found multi-method approaches (geographical information systems, participatory research methods, participatory snowballing, and peer interviewing) to data collection successful in gaining access to parents who did not use Sure Start services. For the founders study, the researcher used snowballing in addition to gatekeepers to recruit potential participants. Lim and colleagues' (2006) internet study of Asian American cancer patients used strategies that included internet groups, clinics, community centers, cultural centers, and community consultants.

Inviting past participants to be part of the new project can be yet another strategy in increasing traditionally underrepresented groups. A growing body of literature is supporting the theoretical concept of "foot-inthe-door" in which people are more likely to participate if they had previously participated (Sadler et al. 2010). Sadler and associates (2010) found participation in a minimally invasive study helps to increase Asian American women in other studies. The "foot-in-the-door" concept can also be relevant for social work practice to get potential clients or community members by first recruiting them for non-stigmatizing services and eventually inform them about other services.

Ethnic matching is another strategy found in the literature in accessing and working with ethnic populations. Ethnic match can increase participant recruitment if the target population feels that they can trust the recruiter (Mouton et al. 1997). Trust can also facilitate the assessment or interview process to increase self-disclosure (Marin and Marin 1991). If the team member is bilingual, ethnic match may help with overcoming language barriers. Sue and Dhindsa (2006) found ethnic match between therapist and client related to length of mental health treatment. On the other hand, the social distance provided by an outsider can "facilitate disclosure more than interaction with someone who is a member of the same community because of a greater threat to privacy and confidentiality" (Neufeld et al. 2001: 586).

\section{Individual Strategies to Increase Access}

During the engagement, participants'/clients' past memories that have been suppressed can be brought up again; social workers need to be prepared to address this issue. Social workers who are focused on research or community work could have a list of appropriate practitioners for referrals. Studies have found ethnic minorities to prefer practitioners of the same ethnicity (Atkinson 1985, Sue 1998). Therefore, when working with ethnic communities, the list of referrals should include practitioners of the same ethnicity as participants. In the founders study, a list of qualified 
clinicians who were Asian American was developed as part of the IRB process in case participants needed to be referred. In the case of former URMs, all had experienced severe trauma during their childhood. The interviewer used open ended questions to allow the participant the option to give as little or as much detail about their past trauma as they chose. Great care was used when asking follow-up questions around topics that included traumatic memories, and probes were generally used to guide the conversation forward when participants appeared in distress. A short debriefing followed each interview as the participant was also given a list of local resources if they felt vulnerable as a result of the interview. Social workers can follow up at the end of any engagement to help add closure to previous work that may have brought up suppressed memories (Anderson et al. 2000).

Partnering with the ethnic community may be time-consuming and labor intensive but it is a common strategy used by many (UyBico et al. 2007) that can help to overcome challenges of mistrust. It is common for research teams and service providers to enter minority communities with a fully developed project and leave upon its completion. That common practice may reinforce feelings of exploitation and mistrust. A better approach may be formation of collaboration with the community of interest throughout all stages of the project to build trust and community ownership for project support (Coleman et al. 1997). The collaboration will help social workers to define the problem congruent to the community's perspective, particularly around stigmatizing issues. Collaboration also checks the social workers' assumptions about the community as well as help to make the project culturally appropriate. For research projects, collaboration will check the validity of the data and its findings. Social workers must present the value of the project as well as possible benefits to the community. Social workers must give back to the community by the work truly benefitting the community and by providing services or participating in the improvement of the community. Researchers can also give back by increasing the community's access to services by making referrals or educating the community about the resources available through mainstream services.

Practitioners who wish to engage with specific communities can also develop an advisory board. A community advisory board may perform as advisors, advocates, gatekeepers, and/or cultural interpreters. Members of the board should be knowledgeable about the community as well as its cultural beliefs, values, language, and history (Strauss et al. 2001). This group can be a good source of opinion and brain-storming as for what may or may not work. Advisory members can consult on ethical issues by placing them within the sociocultural context specific to the community (Yick 2007). The group can assist social workers to make sure that community interests are represented. And can be advocates for the project. As advocates, advisory members will improve the community's awareness of the project as well as reduce stigma of issues in the community. The group can advise on the development of any materials. If leaders of the ethnic community are included in the advisory group, they can help to 
mobilize the community. Uses of community leaders who are trustworthy as spokespeople have found to be successful in public health programs (Jernigan and Wright 1996). Finally, the community advisory group can help disseminate knowledge gained or share information from the project.

Working with the community means doing preliminary work in order to gain knowledge that may influence the success of the project- transportation patterns or cultural beliefs. Social workers must spend time in the community to know who are the key community stakeholders to bring together to achieve the goals of the project. This familiarization process can also help to increase social workers' cultural understanding. Social workers must also spend the time necessary to build and maintain relationships with community stakeholders. This is time consuming because stakeholders may have different perspectives and agendas. There is no substitution for being in the community and building the necessary relationships. In planning to meet with community members, social workers should go to the community rather than having community members come to the social worker's office.

Part of the collaboration also includes social workers maintaining a presence in the community to learn about the culture as well as lending their expertise in matters with which the community may be struggling. Social workers may benefit by working with local establishments. Gaining buy-in of faith-based centers is particularly beneficial if they are trusted by the community (Burnett et al. 2005). Additionally, barbershops and beauty salons, health clubs, restaurants, and ethnic grocery stores can be utilized.

Since much of the research that takes place occurs in association with a university, literature on university-community collaboration is useful. One of the most important challenges to overcome in a university-community relationship is the imbalance of power. Fisher and colleagues (2004) note that often the research agenda controls the process while the community partner simply serves as the venue. They note that the university "...must be able to reimagine its relationship to knowledge production, learning and the community" (Fisher et al. 2004: 32). Communication between universities and community partners is key, particularly around listening on the part of the university (Arbuckle and DeHoog 2004). These collaborations ought to be viewed as mutual learning opportunities, a venue for fostering an ongoing interchange of ideas.

\section{Structural Strategies to Increase Access}

The western model of service delivery or the concept of research and mental health may be new to some immigrant and refugee groups. One strategy is to have a cultural broker to explain them in a way that is understandable and makes sense to the immigrant and refugee group. The cultural broker should be of the same ethnic background so that he or she is seen as an insider (Chen et al. 2005) because cautious immigrants may trust individuals who can relate to them. Additionally, rather than focusing on the name of something, describe what it is. 
Whether it is for research or service, every effort must be taken to reduce burden as a means to overcome some of the barriers ethnic minorities face. Providing reimbursement for inconveniences that includes transportation and babysitting can be helpful. Flexible hours should be employed to include individuals who work nonconventional hours. In the URM study, the researcher allowed the participant to select the time and location of the meeting because it would allow for most convenience as well as security for the participant. In the founders study where participants had limited time availability and were geographically distanced from the researcher, telephone interviews took place. With improved technology, researcher and practitioners may be able to use video conferencing in place of face-to-face interactions if necessary. For research, monetary compensation can be provided if participation would mean being away from employment, particularly for those earning an hourly salary. Depending on the topic of the research, services can also be provided during or after the project.

Social workers need to recognize the complexity of working with ethnic communities (Feldman et al. 2003). This complexity often translates to the time-consuming nature of the project. Planning should take into account and recognize the value of community partners' and participants' time and scheduling constraints. In the founders study, the researcher had to limit the length of the study for practical reasons and eventually came to realize much more planning needed to be done prior to the beginning of the study. In the study with URMs, time to build relationships from the top down ended up extending the schedule of the research project, but was a central consideration for successful completion. Access strategies should be evaluated throughout the project and revised as necessary. Social workers may learn of sensitive areas that need further consideration. When met with challenges recruiting participants, Raghallaigh and Galligan (2010) used an observation period to become more culturally aware of the context in which these challenges were present, and to continue building relationships with potential participants.

While both of the example studies in this article do not require retention rates, for some studies, projects, and services, it may be important for social workers to have a high rate of retention. Based on our work with immigrants and refugees, several strategies can be employed. Obtaining alternative contact information such as friends' or relatives' phone numbers can help locate the individual if he or she relocates or could not be reached for some reason. For continued engagement during long breaks, checking up with the individual through mail or telephone calls can be used. This can be as simple as a reminder as to when individuals need to be engaged with the project again. If appointment reminders are done via the telephone, social workers should make the call or be available to get on the call in case individuals have any questions or are hesitant in continuing their involvement. Engagement of the individual should be flexible and done at a time and place that is most convenient for them. Finally, if individuals are to be compensated for their time, that compensation should be broken up 
throughout the project and made clear as to when individuals will receive them.

Often times, access to participants can be gained through existing organizations that already work with the target population. UyBico and associates (2007) found this strategy to be common in the literature. Gaining initial support from management is important in soliciting help from staff members. A handout should be developed for the purposes of gaining support from various levels of the agency. In the URM study, the researcher met with gatekeepers as high up the chain (the state refugee coordinator) as possible in order to be directed to the appropriate agency gatekeeper. The agency director was more likely to work with the researcher if it was suggested by someone in a more powerful position. It is important to be transparent about the research methods and purpose. In order to be transparent about the benefits, a case needs to be made to show how the project will help the participants recruited through the agency. In the project development and planning phase, input from agency personnel should be sought as to how to best recruit participants in terms of times and ways to recruit as well as how to minimize agency operations (Chen et al. 2005). With the URM study, care was taken by the researcher to meet consistently with the agency director and seek input on the process, structure, and content of the project. This not only improved the relevance of the research question in the study, but also increased the buy-in on the part of a key gatekeeper. The research questions not only addressed what the researcher was interested in learning, but also incorporated useful information for the agency as guided by the agency director.

When working with staff members of the organization, social workers should be mindful of the staff's workload and may themselves be overwhelmed with the needs of their clients. Social workers have the obligation to reciprocate for the staff's time and for facilitating the entry process. The researcher in the URM study provided foster care parenting training session to alleviate the staff's time. Another strategy may be that the social worker becomes part of a planning committee or the organization's board where his or her expertise or time can be of assistance. In the founders study, the social worker joined the local ethnic association board and its planning committees of ethnic events throughout the year. Yet another strategy may be that if the organization does not have a licensed social worker, the social worker can provide supervision time so that the organization can have an intern who often is required to be supervised by a licensed social worker. Finally, social workers can help to meet the needs of the clients that the agency is currently not able to meet. In all these strategies, even if the social worker does not have the expertise required to reciprocate, he or she can arrange for others to provide the service to the organization. 


\section{Strategies to Address Institutional Challenges}

When working with ethnic groups, ethical issues must be placed within the sociocultural context of the target population (Yick, 2007). It is the responsibility of the researcher to ensure that the study being conducted will help the target population. Similarly in practice, the professional maintains the ethical responsibility to provide the best possible services. Sterling and Peterson (1999) recommend social workers addressing biases prior to engaging with the ethnic community.

All project team members must be well-trained and culturally sensitive. In the field of social work, there are multiple frames of reference related to culture. Cultural competence and cultural humility are common terms that refer to a social worker's ability to self-evaluate and self-critique the affect their attitudes and beliefs have on their practice with people from diverse cultural backgrounds. Nunez (2000) suggests cross-cultural efficacy, which implies that neither the professional's nor the client's culture is the "...preferred or more accurate view" (Nunez 2000: 1072). For those working with target populations who may include non-English proficient individuals, linguistically competent team members are necessary.

For successful inclusion of ethnic minorities, social workers must allay fears of exploitation. Social workers must be aware of past abuses of ethnic minority populations in American history and research. Throughout the process, social workers need to continue to be particularly mindful of ethical issues when working with ethnic minorities. Benefits and risks of the service or study must be clearly described. Measures should be taken and assurances need to be made about the fact that minorities are not being used as guinea pigs in order to develop treatment intervention or generate knowledge solely for the benefit for the majority population.

The main element of protection is the informed consent that consists of information disclosed is adequate, truthful, and non-offensive; comprehension of the information; participants' choice to whether or not to be involved; participants' freedom to stop participation; adequate capacity for decision making; and requirement of social worker to report abuses according to state laws, protection of the confidentiality of participants (Anderson and Hatton, 2000). Some groups within the ethnic minority populations may need additional steps to achieve informed consent that includes translation.

The issue of confidentiality is especially important in small ethnic communities where everyone knows one another. Steps must be taken to assure that individuals' participation and their records will be kept confidential. As Smith (2009) states: "If refugee respondents are afraid that their answers can be traced back to them, they may refrain from answering honestly or withdraw from the interview altogether" (Smith 2009: 67). Some research suggests that participants may feel more comfortable being interviewed by an outsider who they know they will not see again (Gans 1999), though access is made more difficult as an outsider. 


\section{Conclusion}

Sharing results, regardless of a social worker's role, will continue the momentum of the iterative relationship between practice and research. Practice will guide better and more relevant research questions will be guided by practice; practices and policies will improve as research responds to those challenges, refining the following questions to be addressed.

In conclusion, this paper has sought to shed light on the challenges that social worker researchers and practitioners face when working with hard-toreach populations. Minority groups, including immigrants and refugees make up a large number of social work service recipients. Many challenges are faced by researchers, community organizers, and practitioners with relation to recruitment and outreach. This paper used two relevant examples of studies with hard-to-reach populations and current literature to present challenges, lessons learned, and strategies. We advocate for continued focus on research and practice with these populations in spite of limited funding, cultural barriers, and the time consuming nature of the undertaking.

\section{References}

Alvidrez J, Arean PA (2002) Psychosocial treatment research with ethnic minority populations: Ethnical considerations in conducting clinical trials. Ethnics \& Behavior 12(1): 103-116.

Anderson DG, Hatton DC (2000) Accessing vulnerable populations for research. Western Journal of Nursing Research 22(2): 244.

Arbuckle MB, DeHoog RH (2004) Connecting a university to a distant neighborhood: Three stages of learning and adaptation. Journal of Community Practice 12(3/4): 53-70.

Atkinson DR (1985) A meta-review of research on cross-cultural counseling and psychotherapy. Journal of Multicultural Counseling and Development, 1: 138153.

Bhopal R (1997) Is research into ethnicity and health racist, unsound, or important science? British Medical Journal 314(7096): 1751-6.

Brown DR, Tapcu M (2003) Willingness to participate in clinical treatment research among older African Americans and Whites. Gerontologis 43(1): 6272.

Brugge D, Kole A, Lu W, Must A (2005) Susceptibility of elderly Asian immigrants to persuasion with respect to participation in research. Journal of Immigrant Health 7(2): 93-101.

Burnett M, Genao I, Wong WF (2005) Race, culture, and trust: Why should I take a shot if I'm not sick? Ethnicity Disease 15(2 Suppl 3), S3.

Chen H, Kramer EJ, Chen T, Chung H (2005) Engaging Asian Americans for mental health research: Challenges and solutions. Journal of Immigrant Health 7(2): 109-116.

Coe C, Gibson A, Spencer N, Stuttaford M (2008) Sure start: Voices of the "hardto-reach". Child: Care, Health \& Development 34(4): 447-453.

Coleman EA, Tyll L, La Croix AZ, Allen C, Leveille SG, Wallace JI, Buchner DM, Grothaus L C, Wagner EH (1997) Recruiting African American older 
adults for a community-based health promotion intervention: Which strategies are effective? American Journal of Preventive Medicine 13(Suppl. 2): 51-56.

Derluyn I, Broekaert E (2008) Unaccompanied refugee children and adolescents: The glaring contrast between a legal and a psychological perspective. International Journal of Law and Psychiatry 31(2008): 319-330.

Epstein S (2008) The rise of "recruitmentology": Clinical research, racial knowledge, and the politics of inclusion and difference. Social Studies Sciences 38(5): 801-832.

Feldman M, Bell J, Berger M (2003) Gaining access: A practical and theoretical guide for qualitative researchers. Walnut Creek, CA: Altamira Press.

Fisher R, Fabricant M, Simmons LS (2004) Understanding contemporary university-community connections: Context, practice, and challenges. Journal of Community Practice 12(3/4): 13-34.

Flick U (2006) An introduction to qualitative research, $3^{\text {rd }}$ ed.. Thousand Oaks, CA: Sage.

Gans HJ (1999) Filling in some holes: Six areas of needed immigration research. American Behavioral Scientist 42(9): 1302-1313.

Hanssen I (2004) An intercultural nursing perspective on autonomy. Nursing Ethnics 11(1): 28-41.

Jenkins S (1980) The ethnic agency defined. Social Service Review 54(2): 249-261.

Jernigan DH, Wright PA (1996) Media advocacy: lessons from community experiences. Journal of Public Health Policy 17(3): 306-330.

Jones B, Gray B (1986) Problems in diagnosing schizophrenia and affective disorders among blacks. Hospital and Community Psychiatry 37(1): 61-65.

Leslie LA (1992) The role of informal support networks in the adjustment of Central American immigrant families. Journal of Community Psychology 20(3): 243-256.

Lim H, Lin C, Liu Y, Chee W, Im E (2006) Effective strategies for recruiting of Asian cancer patients in internet research. Studies in Health Technology and Informatics 122(2006): 882.

Marin B, Marin G (1991) Research with Hispanic populations. Thousand Oaks, CA: Sage.

Mouton C, Harris S, Rovi S, Solorzano P, Johnson M (1997) Barriers to black women's participation in cancer trials. Journal of the National Medical Association 89(11): 721-727.

Museus SD, Chang MJ (2009) Rising to the challenge of conducting research on Asian Americans in higher education. New Directions for Institutional Research 2009(142): 95-105. doi:10.1002/ir.299

Neufeld A, Harrison MJ, Hughes KD, Spitzer D, Stewart MJ (2001) Participation of immigrant women family caregivers in qualitative research. Western Journal of Nursing Research 23(6): 575-591.

Nunez AE (2000) Transforming cultural competence into cross-cultural efficacy in women's health education. Academic Medicine 75(11): 1071-1080.

O’Brien R, Kosoko-Lasaki O, Cook C, Kissell J, Peak F, Williams E (2006) Selfassessment of cultural attitudes and competence of clinical investigators to enhance recruitment and participation of minority populations in research. Journal of the National Medical Association 98(5): 674-682.

Raghallaigh MN, Gilligan R (2010) Active survival in the lives of unaccompanied minors: Coping strategies, resilience, and the relevance of religion. Child and Family Social Work 15(2): 226-237.

Roberson N (1994) Clinical trial participation: Viewpoints from racial/ethnic groups. Cancer Supplement 74(9): 2687-2691. 
Sadler GR, Ko CM, Takahashi M, Ching CR, Lee I, Chuang GC, Lee KK (2010) Increasing Asian American women's research participation: The Asian grocery store-based cancer education program. Contemporary Clinical Trials 31(4): 283-288. doi:10.1016/j.cct.2010.03.008

Sheikh A, Halani L, Bhopal R, Netuveli G, Partridge MR, Car J, Griffiths C, Levy M (2009) Facilitating the recruitment of minority ethnic people into research: Qualitative case study of south Asians and asthma. PLoS Med 6(10): e1000148. doi:10.1371/journal.pmed.1000148.

Smith VJ (2009) Ethical and effective ethnographic research methods: A case study with Afghan refugees in California. Journal of Empirical Research on Human Research Ethics 4(3): 59-72.

SterlingYM, Peterson JW (1999) Scientific inquiry, challenges of recruiting minority populations for research. Journal of the Society of Pediatric Nurses 4(4): 172-174.

Strauss RP, Sengupta S, Quinn SC, Goeppinger J, Soaulding C, Kegeles SM, Millett G (2001) The role of community advisory boards: Involving communities in the informed consent process. American Journal of Public Health, 91(12): 1938-1943.

Sue S (1998) Psychotherapeutic services for ethnic minorities: Two decades of research findings. American Psychologist 43(4): 301-308.

Sue S, Dhindsa MK (2006) Ethnic and Racial Health Disparities Research: Issues and Problems. Health Education \& Behavior 33(4): 459-69.

Sue S, Sue L (2003) Ethnic research is good science. In G Bernal, JE Trimble, AK Burlew, FTL Leong (Eds), Handbook of Racial and Ethnic Minority Psychology, 198-207. Newbury Park, CA: Sage.

UyBico SJ, Pavel S, Gross CP (2007) Recruiting vulnerable populations into research: A systematic review of recruitment interventions. Journal of General Internal Medicine 22(6): 852-863.

Yick AG (2007) Role of culture and context: Ethical issues in research with Asian Americans and immigrants in intimate violence. Journal of Family Violence 22(5): 277-285. 